The Astrophysical Journal, 490:564-570, 1997 December 1

(C) 1997. The American Astronomical Society. All rights reserved. Printed in U.S.A.

\title{
A LOWER BOUND ON THE COSMIC BARYON DENSITY
}

\author{
David H. Weinberg, ${ }^{1}$ Jordi Miralda-Escudé, ${ }^{2}$ LARs Hernquist, ${ }^{3,4}$ AND Neal Katz ${ }^{5}$ \\ Received 1997 January 9; accepted 1997 July 11
}

\begin{abstract}
We derive lower bounds on the cosmic baryon density from the requirement that the high-redshift intergalactic medium (IGM) contain enough neutral hydrogen to produce the observed Ly $\alpha$ absorption in quasar spectra. These analytic bounds follow from a key theoretical assumption - that absorbing structures are on average no more extended in redshift space than in real space-which is likely to hold in the gravitational instability picture of the $L y \alpha$ forest, independently of the details of the cosmological model. The other ingredients that enter these bounds are an estimate of (or lower limit to) the intensity of the photoionizing UV background from quasars, a temperature $T \sim 10^{4} \mathrm{~K}$ for the "warm" photoionized IGM that produces most of the Ly $\alpha$ absorption, a value of the Hubble constant, and observational estimates of the mean Ly $\alpha$ flux decrement $\bar{D}$ or, for a more restrictive bound, the distribution function $P(\tau)$ of Ly $\alpha$ optical depths. With plausible estimates of the quasar UV background and $\bar{D}$, the mean decrement bound implies a baryon density parameter $\Omega_{b} \gtrsim 0.0125 h^{-2}$, where $h \equiv H_{0} /\left(100 \mathrm{~km} \mathrm{~s}^{-1}\right.$ $\mathrm{Mpc}^{-1}$ ). A recent observational determination of $P(\tau)$ implies that $\Omega_{b} \gtrsim 0.0125 h^{-2}$ even for a conservative estimate of the quasar UV background, and $\Omega_{b} \gtrsim 0.018 h^{-2}$ for a more reasonable estimate. These bounds are consistent with recent low estimates of the primordial deuterium-to-hydrogen ratio $(\mathrm{D} / \mathrm{H})_{P}$, which imply that $\Omega_{b} \approx 0.025 h^{-2}$ when combined with standard big bang nucleosynthesis. Since the bounds account only for baryons in the warm IGM, their combination with the nucleosynthesis constraint implies that most of the baryons in the universe at $z \sim 2-4$ were distributed in diffuse intergalactic gas rather than in stars or compact dark objects. The $P(\tau)$ bound on $\Omega_{b}$ is incompatible with some recent high estimates of $(\mathrm{D} / \mathrm{H})_{P}$, unless one drops the assumptions of standard big bang nucleosynthesis or abandons the idea that $\operatorname{Ly} \alpha$ forest lines originate in the smooth, large-scale structures of photoionized gas that arise in gravitational instability theories.
\end{abstract}

Subject headings: cosmology: theory — elementary particles — intergalactic medium quasars: absorption lines

\section{INTRODUCTION}

Following the discovery of the first $z>2$ quasar (Schmidt 1965), Gunn \& Peterson (1965) derived a stringent upper bound on the density of uniformly distributed, neutral hydrogen in intergalactic space, by showing that the redshifted Ly $\alpha$ absorption of neutral gas with more than $\sim 10^{-4}$ of closure density would turn quasar spectra virtually black at short wavelengths, contrary to observation. They concluded that the intergalactic medium (IGM) must be highly ionized or extremely rarefied. Within a few years, it became clear that the ubiquitous absorption lines in quasar spectra are predominantly those of intervening neutral hydrogen (Lynds 1971; Sargent et al. 1980), and subsequent studies have shown that these lines significantly depress the mean flux received from high-redshift quasars blueward of the Ly $\alpha$ emission line (Oke \& Korycansky 1982; Steidel \& Sargent 1987; Jenkins \& Ostriker 1991; Press, Rybicki, \& Schneider 1993, hereafter PRS; Zuo \& Lu 1993; Dobrzycki \& Bechtold 1996; Rauch et al. 1997b). Furthermore, it is now recognized that the ambient UV radiation background produced by high-redshift quasars

\footnotetext{
${ }^{1}$ Department of Astronomy, Ohio State University, 174 West 18th Avenue, Columbus, OH 43210; dhw@astronomy.ohio-state.edu.

${ }^{2}$ Department of Physics and Astronomy, University of Pennsylvania, 209 South 33d Street, Philadelphia, PA 19104; jordi@dept.physics. upenn.edu.

${ }^{3}$ University of California Observatories/Lick Observatory, Santa Cruz, CA 95064; lars@ucolick.org.

${ }^{4}$ Presidential Faculty Fellow.

${ }^{5}$ Five College Astronomy Program, University of Massachusetts, LGRC-517, Amherst, MA 01003; nsk@phast.umass.edu.
}

will strongly photoionize gas near the cosmic mean density, so that a small amount of diffuse neutral hydrogen corresponds to a much larger amount of total hydrogen (e.g., Haardt \& Madau 1996, hereafter HM). In this paper, we will argue that matching the observed Ly $\alpha$ absorption leads to interesting lower bounds on the mean baryon density of the universe, which can be derived from quite general assumptions about the state of the absorbing gas.

Recent cosmological simulations suggest that "Ly $\alpha$ forest" lines arise in diffuse, but nonuniform, intergalactic gas and that they therefore represent a phenomenon closely akin to the "Gunn-Peterson effect" (Cen et al. 1994; Petitjean, Mücket, \& Kates 1995; Zhang, Anninos, \& Norman 1995; Hernquist et al. 1996; for related semianalytic modeling see Bi 1993; Bi \& Davidsen 1997; Hui, Gnedin, \& Zhang 1997). Quantitative analyses show that these simulations require a high baryon density in order to reproduce the observed mean opacity of the forest (Hernquist et al. 1996; Miralda-Escudé et al. 1996; Croft et al. 1997; Rauch et al. 1997b; Zhang et al. 1997; see Rauch, Haehnelt, \& Steinmetz 1997a for a closely related argument). For the UV background predicted by HM based on the observed population of quasars, matching the mean opacity estimates of PRS typically requires $B \gtrsim 2$, where

$$
B \equiv \frac{\Omega_{b} h^{2}}{0.0125}=\frac{\eta}{3.4 \times 10^{-10}}
$$

is the baryon density scaled to the fiducial big bang nucleosynthesis estimate of Walker et al. (1991). Here $h \equiv H_{0} /(100$ $\mathrm{km} \mathrm{s}^{-1} \mathrm{Mpc}^{-1}$ ), and $\eta$ is the baryon-to-photon ratio. 
The bounds on $B$ derived in this paper will not be as high as those derived from the simulations, but they have broader applicability because they are not tied to a specific cosmological scenario, and the simplicity of the arguments that form them makes it easier to understand how changes in the theoretical or observational inputs affect the final result. We do appeal to the simulations to motivate our one crucial assumption, that structures with a volume filling factor $f$ in real space have, on average, a filling factor no larger than $f$ in redshift space. This assumption can also be phrased as a requirement that typical Ly $\alpha$ forest absorbers satisfy $X \geq 1$, where

$$
X \equiv \frac{\text { real-space extent }}{\text { redshift-space extent }}=\frac{H(z) d}{\Delta v}
$$

is the ratio of the Hubble flow across an absorber (with line-of-sight extent $d$ ) to its line width $\Delta v$. This assumption would not hold in a model of spatially compact Ly $\alpha$ clouds whose line widths are determined by thermal broadening. However, in the physical picture that emerges from the simulations, the marginally saturated $\left(N_{\mathrm{HI}} \sim 10^{13}-10^{15}\right.$ $\mathrm{cm}^{-2}$ ) absorption lines that dominate the overall absorption usually arise in moderate-overdensity structures that are expanding in proper coordinates but contracting in comoving coordinates (Miralda-Escudé et al. 1996, 1997). The absorption-line widths are determined largely by these coherent internal motions rather than by thermal motions, and the velocity extent of these features is generally no larger than the Hubble flow across them.

Empirical support for the assumption that $X \geq 1$ comes from observations of quasar pairs, which yield a typical transverse coherence scale $\sim 150 h^{-1} \mathrm{kpc}$ for Ly $\alpha$ forest systems at $z \sim 2$ (Bechtold et al. 1994; Dinshaw et al. 1994, 1995). For a typical line width $\Delta v \sim 25 \mathrm{~km} \mathrm{~s}^{-1}$ (Hu et al. 1995), this length scale would imply $X \sim 3 / R$ (for $\Omega=1$ and $z=2$ ), where $R$ is the ratio of transverse extent to line-ofsight extent in real space. If the absorbers are nonspherical, then they are more often intercepted when they are closer to "face on," so $R$ might reasonably exceed 1 on average. However, the absorbers would have to be highly flattened, coherent sheets in order to reproduce both the observed transverse coherence and the observed line widths while having an average $X$ significantly smaller than 1 . The coincidence of absorption along widely separated lines of sight could, in principle, be caused by clustering of Ly $\alpha$ clouds that are much smaller than the separation itself. However, the interpretation of the coherence scale as a physical size rather than as a clustering length is supported by highresolution spectra of the gravitational lens candidate HE 1104-1805, which show that over a transverse separation $\sim 10 h^{-1} \mathrm{kpc}$ there are almost no anticoincident lines and that coincident lines have very similar redshifts and equivalent widths (Smette et al. 1995; see also Smette et al. 1992 and Bechtold \& Yee 1995). There is also no evidence for strong line-of-sight clustering of $\mathrm{Ly} \alpha$ lines at velocity separations corresponding to $\sim 150 h^{-1} \mathrm{kpc}$, though the line-of-sight signal could conceivably be suppressed relative to the transverse signal by line blending.

Rauch \& Haehnelt (1995) used the large transverse coherence scale to argue that the Ly $\alpha$ forest must contain a substantial fraction of all baryons in the universe at high redshift. Our arguments here are different from those of Rauch \& Haehnelt — in particular, we work directly from observed optical depths instead of from a derived $\mathrm{H}$ I column density distribution-but the spirit is similar. In $\S 2$, we consider the lower bound on $B$ that can be obtained from the mean Ly $\alpha$ flux decrement (Oke \& Korycansky 1982) alone. In $\S 3$, we derive a more restrictive lower bound from the distribution of flux decrements (or equivalent optical depths), recently measured from a set of seven Keck High Resolution Echelle Spectrometer (HIRES) spectra by Rauch et al. (1997b). Our analytic approach complements the direct comparison with simulations carried out by Rauch et al. (1997b), which leads to stronger but less general bounds on the baryon density. We discuss implications of our results in $\S 4$.

\section{A LOWER BOUND FROM THE MEAN FLUX DECREMENT}

A uniform IGM with neutral hydrogen density $n_{\mathrm{H} \text { I }}$ produces a Ly $\alpha$ optical depth

$$
\tau_{u}=\frac{\pi e^{2}}{m_{e} c} f_{\alpha} \lambda_{\alpha} H^{-1}(z) n_{\mathrm{HI}},
$$

where $f_{\alpha}=0.416$ is the $\mathrm{Ly} \alpha$ oscillator strength and $\lambda_{\alpha}=1216 \AA$ is the transition wavelength (Gunn \& Peterson 1965). The Hubble parameter at redshift $z$ is

$$
H(z)=H_{0}\left[\Omega_{0}(1+z)^{3}+\left(1-\Omega_{0}-\lambda_{0}\right)(1+z)^{2}+\lambda_{0}\right]^{1 / 2},
$$

where $\lambda_{0}$ is the cosmological constant $\Lambda$ divided by $3 H_{0}^{2}$. For realistic assumptions about the UV background, the IGM is highly photoionized, and the neutral hydrogen density is

$$
n_{\mathrm{HI}}=\frac{n_{\mathrm{H}} n_{e} \alpha(T)}{\Gamma}=\frac{1.16 n_{\mathrm{H}}^{2} \alpha(T)}{\Gamma},
$$

where $\alpha(T)$ is the recombination coefficient at the gas temperature $T, \Gamma$ is the photoionization rate, and $n_{\mathrm{H}}$ is the total hydrogen density. This condition (eq. [5]) enforces balance between destruction of $\mathrm{H}$ I by photoionization and creation by recombination. In gas with $T \gtrsim 10^{5} \mathrm{~K}$, collisional ionization enhances the destruction rate and lowers $n_{\mathrm{H}}$. The mean value of $n_{\mathrm{H}}$ is

$$
\bar{n}_{\mathrm{H}}=1.07 \times 10^{-7}(1+z)^{3} B \mathrm{~cm}^{-3},
$$

with $B$ as defined in equation (1). Equations (5) and (6) assume a hydrogen mass fraction $X=0.76$ and a helium mass fraction $Y=0.24$. For gas at temperature $T_{4} \equiv T /\left(10^{4}\right.$ $\mathrm{K}) \approx 1$, the recombination coefficient is

$$
\alpha(T)=4.2 \times 10^{-13} T_{4}^{-0.7} \mathrm{~cm}^{3} \mathrm{~s}^{-1}
$$

(Abel et al. 1997). Combining equations (3)-(7) yields

$$
\tau_{u}=2.31 \times 10^{-4}(1+z)^{5}\left(1+\Omega_{0} z\right)^{-1 / 2} h^{-1} T_{4}^{-0.7} \Gamma_{-12}^{-1} B^{2},
$$

where $\Gamma_{-12} \equiv \Gamma /\left(10^{-12} \mathrm{~s}^{-1}\right)$ and we have assumed $\Lambda=0$ to compute $H(z)$. Equation (8) agrees with, e.g., equation (36) of HM.

The mean Ly $\alpha$ flux decrement produced by this uniform medium is $\bar{D} \equiv\left\langle 1-e^{-\tau}\right\rangle=1-e^{-\tau_{u}} \equiv D_{u}$. If the medium is optically thin (i.e., $\tau_{u} \ll 1$ ), then clumping the gas tends to increase $\bar{D}$, because the mean neutral fraction at fixed temperature increases in proportion to $\left\langle n_{\mathrm{H}}^{2}\right\rangle /\left\langle n_{\mathrm{H}}\right\rangle^{2}$. However, once the gas clumps produce absorption lines with optical depths $\gtrsim 1$, then $\bar{D}$ is decreased by further clumping, because more neutral atoms are added to saturated regions 
(where they cannot contribute to increasing $\bar{D}$ ), at the expense of the interclump medium, where the resulting absorption must decrease as matter is moved into the clumps.

Consider an idealized case in which all the gas collects into nonoverlapping, uniform-density clumps that are not necessarily spherical, which have a volume filling factor $f$ and, thus, an overdensity of $1 / f$. If a clump has the same extent in redshift space as it does in real space, then it produces an absorption line with optical depth $\tau_{c}=\tau_{u} / f^{2}$ and a flux decrement of $D_{c}=1-e^{-\tau_{c}}$. Since these lines fill a fraction $f$ of the spectrum, the mean flux decrement is

$$
\bar{D}=f D_{c}=f\left(1-e^{-\tau_{u} / f^{2}}\right) .
$$

In the optically thin limit, $\tau_{u} / f^{2} \ll 1$, a Taylor expansion of equation (9) yields $\bar{D}=D_{u} / f$. In the saturated line limit $\tau_{u} / f^{2} \gg 1$, and equation (9) yields $\bar{D}=f$. Thus, if $\tau_{u} \ll 1$, clumping the gas (lowering $f$ ) initially increases the absorption but decreases it once lines become saturated, as expected from our argument above.

It is straightforward to show (by setting $d \bar{D} / d f=0$ ) that the maximum value of $\bar{D}$ in equation (9) occurs when the optical depth of the clumps is $\tau_{u} / \hat{f}^{2}=\hat{\tau}_{c}=1.25643 \ldots ; \hat{\tau}_{c}$ is the solution to the equation $\hat{\tau}_{c}=\frac{1}{2}\left(e^{\hat{\tau}_{c}}-1\right)$. If $\tau_{u}>\hat{\tau}_{c}$, then clumping can only decrease the overall absorption, and $D_{u}$ is the maximum value of the mean flux decrement. If $\tau_{u}<$ $\hat{\tau}_{c}$, then the maximum decrement occurs for filling factor

$$
\hat{f}=\left(\tau_{u} / \hat{\tau}_{c}\right)^{1 / 2}=0.89214 \tau_{u}^{1 / 2},
$$

implying a maximum decrement

$$
\bar{D}_{\max }=\hat{f}\left(1-e^{-\hat{\tau}_{c}}\right)=0.71533 \hat{f}=0.63818 \tau_{u}^{1 / 2} .
$$

This maximum decrement applies to any model with an arbitrary distribution function of the gas density, because the general case can be treated as a superposition of cases in which all the gas is in regions of constant density. Thus, the average absorption cannot exceed that of this optimal uniform-clump case.

For an observed value of $\bar{D}$, equations (8) and (11) can be combined to obtain a lower bound on $B$ :

$$
B_{\min }=\left\{\begin{array}{cc}
65.8(\bar{D} / 0.63818)(1+z)^{-5 / 2}\left(1+\Omega_{0} z\right)^{1 / 4} \\
\times h^{1 / 2} T_{4}^{0.35} \Gamma_{-12}^{1 / 2}, \quad \text { for } \bar{D} \leq 0.71533, \\
65.8[-\ln (1-\bar{D})]^{1 / 2}(1+z)^{-5 / 2}\left(1+\Omega_{0} z\right)^{1 / 4} \\
\times h^{1 / 2} T_{4}^{0.35} \Gamma_{-12}^{1 / 2}, \quad \text { for } \bar{D} \geq 0.71533
\end{array}\right.
$$

The latter equation applies when $\bar{D}>1-e^{-\hat{\tau}_{c}}$, so that the uniform IGM is the optimal case. Equation (12) yields a lower bound on the baryon density on the assumption that absorbing regions have, on average, the same filling factor in redshift space as in real space.

If one assumes more generally that the absorbing regions have a constant ratio $X$ of real-space extent to redshiftspace extent, then the optical depth of the clumps changes to $\tau_{c}=\tau_{u} X / f^{2}$, and the spectral filling factor to $f / X$ (or to unity, if $f>X$ ). One can carry through the same reasoning as above to find that the bounds in equation (12) simply change by the factor $X^{1 / 2}$. As discussed in $\S 1$, cosmological simulations and quasar pair observations suggest that $X \gtrsim$ 1 for systems that dominate the mean absorption, making equation (12) conservative. Models with thermally broadened or expanding clouds could have $X<1$ and thereby escape the bound derived in equation (12), but the absorbers must be inflated by a factor of 4 in redshift space in order to weaken the bound by a factor of 2 .

In order to obtain numerical values for $B_{\min }$, we must adopt values of the mean flux decrement $\bar{D}$, the photoionization rate $\Gamma_{-12}$, the gas temperature $T_{4}$, and the cosmological parameters $\Omega_{0}$ and $h$. PRS, using the high-redshift quasar data of Schneider, Schmidt, \& Gunn (1994), found $\bar{D}(z)=1-e^{-\tau_{\text {eff }}(z)}$ with $\tau_{\text {eff }}(z)=0.0037(1+z)^{3.46}$. The absorption data used to derive this fit cover the redshift range $2.5<z<4.2$. When extrapolated to $z=2$, this formula yields $\bar{D}=0.15$, in agreement with the value derived by Rauch et al. (1997b) from Keck HIRES spectra. Based on emission from the observed population of quasars and reprocessing by the Ly $\alpha$ forest, HM found $\Gamma_{-12}=$ 1.4-1.6 for $2<z<3$, and this value agrees well with recent estimates from the Ly $\alpha$ forest proximity effect (Giallongo et al. 1996). In cosmological simulations with a photoionizing background, Katz, Weinberg, \& Hernquist (1996) found $T_{4} \approx 0.6$ for gas at the cosmic mean density. The solid line in Figure 1 shows the lower bound $B_{\min }$ obtained using the PRS formula for $\bar{D}(z), \Gamma_{-12}=1.4, T_{4}=0.6, h=0.65$, and $\Omega_{0}=0.3$. The derived constraint, $B \gtrsim 1$, is only weakly dependent on redshift in the range $2<z<3$ because the observed dependence of $\bar{D}$ on $z$ is canceled by the redshift factors in equation (12). Our choice of temperature is conservatively low, since overdense gas is usually hotter than gas at the mean density, and since the Katz et al. (1996) simulations do not incorporate heat injection during reionization (Miralda-Escudé \& Rees 1994). Inclusion of reionization heating, the magnitude of which is theoretically uncertain, could raise the gas temperatures enough to increase $B_{\min }$ by $20 \%-50 \%$.

Of the various inputs to the lower bound in equation (12), $B_{\min }$ is most sensitive to the mean flux decrement $\bar{D}$ itself. As

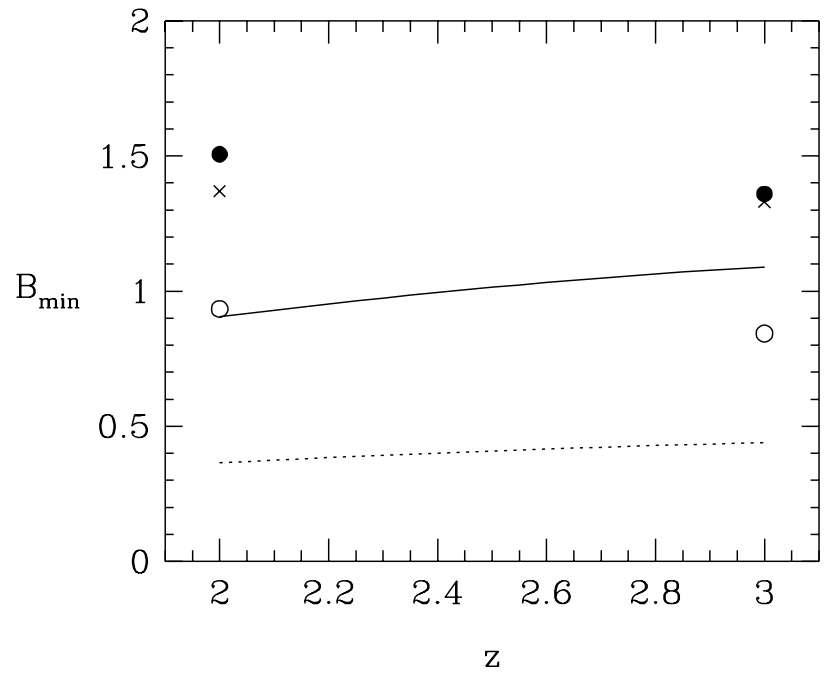

Fig. 1.-Lower bounds on the scaled baryon density $B \equiv \Omega_{b} h^{2} / 0.0125$, obtained from the mean flux decrement (solid and dotted lines, eq. [12]) and from the optical depth distribution (filled and open circles, eq. [22]). The solid line shows the mean decrement bound with the PRS formula for $\bar{D}(z)$, $\Omega_{0}=0.3, h=0.65, T_{4}=0.6$, and $\Gamma_{-12}=1.4$. The dotted line shows the mean decrement bound for conservative parameter choices: $\bar{D}(z)$ equal to $65 \%$ of the PRS values, $h=0.5$ and $\Gamma_{-12}=0.7$. Filled circles show the $P(\tau)$ bounds at $z=2$ and $z=3$, using $\beta=0.633, \Omega_{0}=0.3, h=0.65, \bar{T}_{4}=0.6$, and $\Gamma_{-12}=1.4$. Open circles show the corresponding bounds for $h=0.5$ and $\Gamma_{-12}=0.7$. Crosses show Rauch et al.'s (1997b) estimates of $B$ for $h=0.5$, and $\Gamma_{-12}=0.7$, obtained by comparing the observed optical depth distribution with that derived from a numerical simulation of the standard cold dark matter model. 
already mentioned, the value obtained by Rauch et al. (1997b) at $z=2$ agrees almost perfectly with the PRS formula that we have used. At $z=3$, the PRS formula predicts $\bar{D}=0.36$, and Rauch et al. (1997b) measure $\bar{D}=0.32$, which rises to $\bar{D}=0.35$ after including a theoretically estimated correction for continuum-fitting bias. Steidel \& Sargent (1987) found $\bar{D}=0.24$ for a sample of seven quasars with a mean absorption redshift of 2.64; the PRS formula yields $\bar{D}=0.28$ at this redshift. These three studies thus agree to $15 \%$ or better. However, Zuo \& Lu (1993) and Dobrzycki \& Bechtold (1996) found values of $\bar{D}$ that are typically about $35 \%$ smaller than the PRS values. The Rauch et al. (1997b) values seem the most secure because they are based on the highest quality quasar spectra, but the sample used is relatively small, so further analyses of spectra with similar resolution and signal-to-noise ratio will be needed to arrive at a definitive measurement.

The other significant uncertainty in $B_{\min }$ is the value of $\Gamma_{-12}$. The HM estimate is more likely to be too low than too high since it assumes that quasars are the only source of the UV background. However, HM did include an extrapolation of the quasar luminosity function to allow for faint sources below existing survey limits, and Rauch et al. (1997b) conclude that $\Gamma_{-12}$ could be up to a factor of 2 below HM's estimate, at the price of worsening the agreement with proximity-effect estimates (e.g., Bajtlik, Duncan, \& Ostriker 1988; Bechtold 1994; Giallongo et al. 1996; Cooke, Espey, \& Carswell 1997). The dotted line in Figure 1 shows the value of $B_{\min }$ obtained after reducing $\bar{D}(z)$ to $65 \%$ of the PRS values, lowering $\Gamma_{-12}$ to 0.7 , and, for good measure, dropping $h$ to 0.5 . With all of the parameters in equation (12) pushed to these favorable values, the lower bound is $B_{\min } \approx 0.45$. Of course, achieving the observed absorption with $B=B_{\min }$ requires that all of the baryons be collected into uniform-density, $6000 \mathrm{~K}$ gas clumps that have $\tau=\hat{\tau}_{c}$, which further requires that the overdensity of these clumps evolve with redshift as $1 / f=0.71533 / \bar{D}(z)$ (see eq. [11]).

\section{A LOWER BOUND FROM THE OPTICAL DEPTH DISTRIBUTION}

The clustering pattern required to achieve the bound of equation (12) is not just physically contrived but is also inconsistent with the observed properties of quasar spectra. These indicate absorption at a range of optical depths, not a set of $\tau=1.25$ lines separated by absorption-free regions. The two cosmological simulations discussed in Rauch et al. (1997b) produce spectra whose optical depth distributions agree well with observations, and Rauch et al. argue that they therefore provide reliable models of the IGM with which to derive $B$, given values of $\Gamma_{-12}$ and $h$. Here we extend the argument of $\S 2$ to derive a lower bound on $B$ directly from the observed optical depth distribution, based on some simple assumptions about the IGM.

If we ignore peculiar velocities and thermal broadening, then the optical depth of gas with overdensity $n_{\mathrm{H}} / \bar{n}_{\mathrm{H}}$ and temperature $T$ is

$$
\tau=\tau_{u}\left(\frac{n_{\mathrm{H}}}{\bar{n}_{\mathrm{H}}}\right)^{2} \frac{\alpha(T)}{\alpha\left(T_{u}\right)},
$$

where $\tau_{u}$ and $T_{u}$ are, respectively, the optical depth and temperature that the gas would have if uniformly distributed. The recombination coefficient is $\alpha(T) \propto T^{-0.7}$ for the temperature range of interest. If we assume a relation between density and temperature, then we can associate a density with each optical depth $\tau$. In cosmological simulations that adopt $B=1$ and the HM background spectrum, Weinberg, Hernquist, \& Katz (1997) find $T_{4} \approx 0.6\left(\rho_{b} / \bar{\rho}_{b}\right)^{0.6}$ for the gas that dominates the absorption, with higher temperatures in collapsed, shock-heated regions. The $T \propto \rho^{0.6}$ relation arises because denser gas absorbs energy from the photoionizing background more rapidly (see further discussion by Croft et al. 1997; Hui \& Gnedin 1997; MiraldaEscudé et al. 1997). Reionization heating (Miralda-Escudé $\&$ Rees 1994) can raise the temperature of the gas independently of density and thus tends to produce a higher multiplicative constant and a weaker trend with density. We will assume that

$$
T_{4}=\bar{T}_{4}\left(n_{\mathrm{H}} / \bar{n}_{\mathrm{H}}\right)^{\gamma}
$$

which implies that

$$
n_{\mathrm{H}}=\bar{n}_{\mathrm{H}}\left(\tau / \tau_{u}\right)^{\beta}, \quad \beta \equiv(2-0.7 \gamma)^{-1} .
$$

Theoretically plausible ranges at $z=2-3$ are $T_{4} \sim 0.5-1.5$ and $\gamma \sim 0.0-0.6(\beta \sim 0.5-0.65$; see Hui \& Gnedin 1997). Since shocked gas is heated to higher temperatures and produces less absorption, the assumption that all of the gas follows the temperature-density relation tends to underestimate the amount of baryons present.

Let $P(\tau)$ denote the optical depth probability distribution, so that $P(\tau) d \tau$ is the probability that a randomly selected point on a spectrum has optical depth in the range $\tau$ to $\tau+d \tau$. We can use equation (15) to compute the mean density by integrating over $P(\tau)$ :

$$
\bar{n}_{\mathrm{H}}=\int_{0}^{\infty} n_{\mathrm{H}}(\tau) P(\tau) d \tau=\bar{n}_{\mathrm{H}} \tau_{u}^{-\beta} \int_{0}^{\infty} \tau^{\beta} P(\tau) d \tau,
$$

and thus

$$
\tau_{u}=\left[\int_{0}^{\infty} \tau^{\beta} P(\tau) d \tau\right]^{1 / \beta}
$$

where $\tau_{u}$ depends on $B$ and other parameters as indicated in equation (8). We have relied on our assumption of Hubble flow broadening both to associate a physical density with a spectral optical depth and to identify a probability $P(\tau) d \tau$ in redshift space with an equal filling factor in real space. We can crudely incorporate a more general model by assuming that the ratio $X$ of real-space extent to redshift-space extent is a function of $\tau$ alone, $X=X(\tau)$. If $X(\tau)>1$, then "squeezing" in redshift space has enhanced the optical depth by a factor $X(\tau)$, but the spectral filling factor of $P(\tau) d \tau$ corresponds to a larger real-space filling factor of $X(\tau) P(\tau) d \tau$. Thus

$$
\bar{n}_{\mathrm{H}}=\int_{0}^{\infty} n_{\mathrm{H}}(\tau) P(\tau) d \tau=\bar{n}_{\mathrm{H}} \tau_{u}^{-\beta} \int_{0}^{\infty}[\tau / X(\tau)]^{\beta} X(\tau) P(\tau) d \tau,
$$

implying that

$$
\tau_{u}=\left\{\int_{0}^{\infty}[X(\tau)]^{1-\beta} \tau^{\beta} P(\tau) d \tau\right\}^{1 / \beta} .
$$

Conceptually, it is helpful to rewrite this equation in the form

$$
\tau_{u}=X_{w}^{(1-\beta) / \beta}\left[\int_{0}^{\infty} \tau^{\beta} P(\tau) d \tau\right]^{1 / \beta}
$$


where $X_{w}$ is the value of $X$ weighted by its contribution to the integral in equation (19):

$$
X_{w} \equiv\left\{\frac{\int_{0}^{\infty}[X(\tau)]^{1-\beta} \tau^{\beta} P(\tau) d \tau}{\int_{0}^{\infty} \tau^{\beta} P(\tau) d \tau}\right\}^{1 /(1-\beta)} .
$$

In the picture suggested by cosmological simulations, $X(\tau)$ is typically smaller than unity for small $\tau$, since the lowest optical depths arise primarily in underdense regions that are expanding faster than the Hubble flow. It then rises above unity for intermediate $\tau$, where the gas is expanding more slowly than Hubble flow as it falls into overdense structures. Saturated absorption lines are often thermally broadened even in cosmological simulations, so $X(\tau)$ may fall below unity again at high $\tau$. Averaging over these three regimes, $X_{w} \approx 1$ is a fair approximation for gravitational instability models of the Ly $\alpha$ forest (see below). Values of $X_{w} \ll 1$ would require dense clouds that were thermally broadened or expanding rapidly relative to Hubble flow.

We can combine equation (20) with equation (8) for the uniform optical depth to obtain a new lower bound on $B$ :

$$
\begin{aligned}
B_{\min }= & 65.8 X_{w}^{(1-\beta) / 2 \beta}\left[\int_{0}^{\infty} \tau^{\beta} P(\tau) d \tau\right]^{1 / 2 \beta} \\
& \times(1+z)^{-5 / 2}\left(1+\Omega_{0} z\right)^{1 / 4} h^{1 / 2} \bar{T}_{4}^{0.35} \Gamma_{-12}^{1 / 2} .
\end{aligned}
$$

If all baryons were in the form of intergalactic gas that followed the temperature-density relation (eq. [14]), then equation (22) would be an estimate of the baryon density rather than a lower bound. However, some fraction of the baryons should be in stars, in shock-heated gas, and (possibly) in baryonic dark matter. Furthermore, since it is difficult to measure the optical depth once $e^{-\tau}$ approaches zero, we will in practice have to compute the integral in equation (22) with a conservative assumption, e.g., that all regions with $\tau>\tau_{\max } \sim 3$ (transmission less than 0.05) have $\tau=\tau_{\max }$. Equation (22) therefore leads to a lower bound on $B$ rather than an estimate.

Note that if we adopt the optimal uniform-density clump model from $\S 2$, then $P(\tau)=\hat{f} \delta\left(\hat{\tau}_{c}\right)+(1-\hat{f}) \delta(0)$, where $\delta(x)$ denotes the Dirac delta function. With $\beta=\frac{1}{2}$, the integral in equation (22) becomes $\hat{\tau}_{c}^{1 / 2} \hat{f}$, which, by equation (11), is $\hat{\tau}_{c}^{1 / 2} \bar{D} /\left(1-e^{-\hat{\tau}_{c}}\right)=\bar{D} / 0.63818$. With $X_{w}=1$, we recover the bound (eq. [12]) from $\S 2$, as expected. The maximum value of $\left(1-e^{-\tau}\right) / \tau^{1 / 2}$ occurs when $\tau=\hat{\tau}_{c}$, so for any other optical depth distribution that has the same mean decrement $\bar{D}=\int_{0}^{\infty}\left(1-e^{-\tau}\right) P(\tau) d \tau$, the factor $\int_{0}^{\infty} \tau^{1 / 2} P(\tau) d \tau$ must exceed $\bar{D} / 0.63818$, and equation (22) yields a more restrictive lower bound on $B$ than equation (12). This derivation from the optical depth probability distribution is an alternative route to equation (12).

We have checked that equation (22) with $X_{w}=1$ yields a lower bound to $B$ in realistic models of the Ly $\alpha$ forest by applying it to spectra from Croft et al.'s (1997) simulations of three cold dark matter cosmological models with $B=1$. We measure $P(\tau)$ from the simulations at $z=2$ and $z=3$, in each case using the value of $\Gamma_{-12}$ required to match the PRS mean flux decrement. [See Fig. 11 of Croft et al. 1997 for plots of $P(\tau)$ at $z=2.33$.] We then apply equation (22), assuming that all regions with $\tau>3$ have $\tau=3$ in order to account for the limited ability of realistic data to estimate optical depths in saturated regions. The derived lower bound is about $70 \%$ of the models' true baryon density in the "standard" (SCDM; $\Omega=1, h=0.5, \sigma_{8}=0.7$ ) and open $\left(\mathrm{OCDM} ; \Omega_{0}=0.4, h=0.65, \sigma_{8}=0.75\right)$ models and about
$50 \%$ of the true baryon density in the COBE-normalized $\Omega=1$ model (CCDM; $\Omega=1, h=0.5, \sigma_{8}=1.2$ ). The bounds are insensitive to the value of $\tau_{\max }$, changing by at most $5 \%-10 \%$ if $\tau_{\max }$ is changed to 2 or to 4 . The baryons "missed" by equation (22) are those that have been shockheated to high temperatures, condensed into very high density clumps with $\tau \gg 3$ (giving rise to Lyman limit and damped Ly $\alpha$ systems), or converted into stars. These are a larger fraction of the total baryon density in the CCDM model because of its higher mass fluctuation amplitude. For the SCDM model, we have estimated $X_{w}$ directly by comparing the derived values of $B_{\min }$ with values derived from spectra that include no thermal broadening or peculiar velocity distortions (and thus have $X_{w}=1$ by construction). We find $X_{w}=1.15$ at $z=2$ and $X_{w}=1.35$ at $z=3$, so while infall (in comoving coordinates) leads to $X_{w}>1$ as expected, assuming $X_{w}=1$ only reduces $B_{\min }$ by $4 \%$ at $z=2$ and $9 \%$ at $z=3$ in this model.

Rauch et al. (1997b) have measured the cumulative distribution of Ly $\alpha$ flux decrements from Keck HIRES spectra of seven quasars, whose emission redshifts range from 2.5 to 4.5. They find that artificial spectra from the SCDM simulation mentioned above reproduce the observed flux decrement distribution quite accurately, once $\Gamma_{-12}$ is chosen so that the mean decrement matches that derived from the data. The artificial (and real) spectra include noise and are measured with a locally estimated continuum level. Since we would like to use the true optical depth distribution, free of observational artifacts, to obtain $B_{\min }$, we measure $P(\tau)$ directly from the noiseless, simulated spectra and use it as a surrogate for the observed $P(\tau)$ in equation (22). As before, we set $\tau=3$ in the regions where $\tau>3$. These high optical depth regions cover $9 \%$ of the spectrum at $z=3$ and $2.5 \%$ at $z=2$.

The filled circles in Figure 1 show the bound $B_{\text {min }}$ obtained from equation (22) at $z=2$ and $z=3$, assuming $X_{w}=1, \beta=0.633$ (i.e., $\gamma=0.6$ ), $\Omega_{0}=0.3, h=0.65, \bar{T}_{4}=$ 0.6 , and $\Gamma_{-12}=1.4$. These assumptions correspond to those used to obtain the solid line, and as expected, the values of $B_{\min }$ derived from the optical depth distribution are higher than those derived from the mean flux decrement alone. At $z=3$ the bound increases by about $25 \%$ to $B_{\min }=1.35$, and at $z=2$ it increases by nearly $70 \%$ to $B_{\min }=1.5$. The open circles indicate the bounds derived for $h=0.5$ and $\Gamma_{-12}=0.7$ (with other parameters unchanged): $B_{\min }=0.85$ at $z=3$ and $B_{\min }=0.95$ at $z=2$. These are substantially stronger than the mean decrement bounds represented by the dotted line, which also assume $h=0.5$ and $\Gamma_{-12}=0.7$. However, in moving from the solid line to the dotted line we reduced the mean flux decrements by $35 \%$, while the filled and open circles are both based on the Rauch et al. (1997b) measurements of the optical depth distribution. If these measurements are accurate (the small sample size being their primary limitation), then the mean baryon density cannot be much smaller than $B \sim 0.9$ unless $\Gamma_{-12}$ is smaller than $0.7, h$ is smaller than 0.5 , or $\operatorname{Ly} \alpha$ absorbers are substantially larger in redshift space than in real space, so that $X_{w}<1$. The crosses in Figure 1 show the values of $B$ that Rauch et al. (1997b) obtain by requiring that the SCDM simulation match the observed $P(\tau)$ with $\Gamma_{-12}=0.7$ and $h=0.5$. These lie about a factor 1.5 above the open circles because the direct comparison to the simulation allows these estimates to include the contribution of stars and shock-heated gas. 


\section{DISCUSSION}

The bound on the baryon density from the mean flux decrement (equation [12]) and the bound from the optical depth distribution (equation [22]) are the principal results of this paper. The mean decrement bound leads to $B \gtrsim 1$ if one takes the most convincing estimates of $\bar{D}$ and of the photoionization rate $\Gamma_{-12}$ from quasars. With the Rauch et al. (1997b) determination of $P(\tau)$, the optical depth distribution bound yields $B \gtrsim 1$ even if one reduces $\Gamma_{-12}$ to half of the best estimate value. These constraints imply a baryonto-photon ratio of $\eta \gtrsim 3.4 \times 10^{-10}$ and, assuming the standard model for big bang nucleosynthesis, a corresponding primordial deuterium-to-hydrogen ratio $(\mathrm{D} / \mathrm{H})_{P} \lesssim 6 \times$ $10^{-5}$. This constraint is consistent with the estimate $(\mathrm{D} / \mathrm{H})_{P}=(2.3 \pm 0.4) \times 10^{-5}$ of Tytler, Fan, \& Burles (1996) from a high-redshift Lyman limit system, but it is inconsistent with the estimates of $(\mathrm{D} / \mathrm{H})_{P} \gtrsim 10^{-4}$ obtained from other high-redshift Lyman limit systems by Songaila et al. (1994), Carswell et al. (1994), and Rugers \& Hogan (1996a, 1996b). Current observational estimates of $P(\tau), \Gamma_{-12}$, and $h$ can only be reconciled with the high $(\mathrm{D} / \mathrm{H})_{P}$ estimates by abandoning the gravitational instability picture of the Ly $\alpha$ forest and returning to a scenario of dense, thermally broadened clouds with $X_{w}<1$ or, more radically, by abandoning standard big bang nucleosynthesis.

The analytic arguments presented in this paper complement Rauch et al.'s (1997b) direct comparison between simulations and observations in several ways. They show that the requirement of a high baryon density follows essentially from one very general result of the simulations, namely, that the Ly $\alpha$ forest lines are broadened primarily by residual Hubble expansion rather than by thermal motions. Changes to the cosmological parameters or improvements in the numerical resolution of the simulations could only erase the high- $\Omega_{b}$ requirement if they altered a basic physical property of this model of the Ly $\alpha$ forest, the linebroadening mechanism. It is unlikely that such a change could happen without destroying the current good agreement between cosmological simulations and observations of the Ly $\alpha$ forest (as documented in Cen et al. 1994; Zhang et al. 1995, 1997; Hernquist et al. 1996; Miralda-Escudé et al. 1996; Davé et al. 1997). While Rauch et al. (1997b) only analyze simulations of two cosmological models, the expression of $B_{\min }$ directly in terms of $P(\tau)$ in equation (22) strengthens Rauch et al.'s argument that any alternative cosmological model that reproduces the observed optical depth distribution will also require a high baryon density. When combined with Hui \& Gnedin's (1997) technique for computing $\bar{T}_{4}$ and $\beta$, equation (22) also offers a straightforward way to examine the effect of different reionization histories or UV background spectra on the $\Omega_{b}$ bounds. Finally, as future observations yield new determinations of $\bar{D}$ or $P(\tau)$, equations (12) and (22) can be used to convert these measurements into lower bounds on $\Omega_{b}$ that depend on a few simple parameters describing the IGM and the background cosmology.

Given an estimated value of $\Gamma_{-12}$, equation (22) provides an estimate of the number of baryons in the warm
$\left(T \sim 10^{3}-10^{5} \mathrm{~K}\right)$, diffuse $(\rho / \bar{\rho} \lesssim 10)$ intergalactic medium. With the Rauch et al. (1997b) $P(\tau)$, HM's value $\Gamma_{-12}=1.4$, and plausible choices for other parameters (see Fig. 1 legend), one obtains $B_{\mathrm{IGM}} \approx 1.4$ at $z \sim 2-3$ (Fig. 1, filled circles). Within standard big bang nucleosynthesis, one must generously increase the estimated observational errors in the primordial ${ }^{4} \mathrm{He}$ abundance even to accommodate $B$ as large as 2 (see, e.g., Hata et al. 1995), and measurements of $(\mathrm{D} / \mathrm{H})$ in the local interstellar medium (see, e.g., McCullough 1992; Linsky et al. 1995) imply $B \lesssim 2.4$. This high value of $B_{\text {IGM }}$ therefore suggests that the warm IGM contains most of the baryons in the universe at these redshifts, as predicted by the cosmological simulations (see also Shapiro, Giroux, \& Babul 1994) and as argued by Rauch \& Haehnelt (1995) on different, but physically related, empirical grounds.

While most of the high-redshift hydrogen resides in the warm, photoionized IGM, most of the neutral hydrogen resides in high column density, damped Ly $\alpha$ systems. Because we truncate $P(\tau)$ at $\tau_{\max }=3$, equation (22) severely undercounts the gas in these systems, which contribute $B_{\text {DLA }} \sim 0.1-0.3 h^{2}$ at $z \sim 3$ (Wolfe et al. 1995). Our lower limit to $B_{\mathrm{IGM}}$ far exceeds the baryon density of stars in bright galaxies today, $B \sim 0.16 h^{2}$ (Persic \& Salucci 1992). Thus, the baryons that were in the warm IGM at $z=2$ must either (1) remain in the IGM today, (2) have formed brown dwarfs or another form of baryonic dark matter since $z=2$, or (3) have formed stars in systems of very low surface brightness that have been missed in standard estimates of the galaxy luminosity function.

There are a number of anticipated observational developments that might strengthen (or weaken) one's confidence in the bounds plotted in Figure 1. The most important will be determinations of $\bar{D}$ and $P(\tau)$ from larger samples of highresolution, high signal-to-noise ratio quasar spectra, since the values of $B_{\min }$ depend primarily on these observational inputs. Ongoing quasar surveys and, in a few years, the Sloan Digital Sky Survey will yield improved determinations of the quasar luminosity function, which can be combined with the HM formalism to yield more definitive estimates of the quasar contribution to the photoionizing background. Further analyses of quasar pairs and studies of absorption-line shapes in high-resolution spectra may provide more compelling evidence for extended Ly $\alpha$ forest absorbers that are broadened largely by Hubble flow, as predicted by cosmological simulations. Such observations would support our key theoretical assumption that $X_{w} \gtrsim 1$, and they would reinforce the view that the Ly $\alpha$ forest arises in a smoothly fluctuating intergalactic medium that is the dominant reservoir of high-redshift baryons.

We thank Michael Rauch and Rupert Croft for helpful discussions. We acknowledge support from NASA grants NAG 5-3111 and NAG 5-3525 and from the NSF through grant ASC 93-18185 and the Presidential Faculty Fellows program. Computational support was provided by the San Diego, Pittsburgh, and Illinois Supercomputing Centers. 


\section{REFERENCES}

Abel, T., Anninos, P., Zhang, Y., \& Norman, M. L. 1997, NewA, 2, 181

Bajtlik, S., Duncan, R. C., \& Ostriker, J. P. 1988, ApJ, 327, 570

Bechtold, J. 1994, ApJS, 91, 1

Bechtold, J., Crotts, A. P. S., Duncan, R. C., \& Fang, Y. 1994, ApJ, 437, L83

Bechtold, J., \& Yee, H. K. C. 1995, AJ, 110, 1984

Bi, H. 1993, ApJ, 405, 479

Bi, H., \& Davidsen, D. E. 1997, ApJ, 479, 523

Carswell, R. F., Rauch, M., Weymann, R. J., Cooke, A. J., \& Webb, J. K. 1994, MNRASS, 268, L1

Cen, R., Miralda-Escudé, J., Ostriker, J. P., \& Rauch, M. 1994, ApJ, 437, L9

Cooke, A. J., Espey, B., \& Carswell, R. F. 1997, MNRAS, 284, 552

Croft, R. A. C., Weinberg, D. H., Katz, N., \& Hernquist, L. 1997, ApJ, 488, 532

Davé, R., Hernquist, L., Weinberg, D. H., \& Katz, N. 1997, ApJ, 477, 21

Dinshaw, N., Foltz, C. B., Impey, C. D., Weymann, R. J., \& Morris, S. L. 1995, Nature, 373, 223

Dinshaw, N., Impey, C. D., Foltz, C. B., Weymann, R. J., \& Chaffee, F. H. 1994, ApJ, 437, L87

Dobrzycki, A., \& Bechtold, J. 1996, ApJ, 457, 102

Giallongo, E., Cristiani, S., D’Odorico, S., Fontana, A., \& Savaglio, S. 1996, ApJ, 466, 46

Gunn, J. E., \& Peterson, B. A. 1965, ApJ, 142, 1633

Haardt, F., \& Madau, P. 1996, ApJ, 461, 20 (HM)

Hata, N., Scherrer, R. J., Steigman, G., Thomas, D., Walker, T. P., Bludman, S., \& Langacker, P. 1995, Phys. Rev. Lett.. 75, 3977

Hernquist, L., Katz, N., Weinberg, D. H., \& Miralda-Escudé, J. 1996, ApJ, 457, L5

Hu, E. M., Kim, T. S., Cowie, L. L., Songaila, A., \& Rauch, M. 1995, AJ, 110,1526

Hui, L., \& Gnedin, N. 1997, MNRAS, submitted (astro-ph/9612232)

Hui, L., Gnedin, N., \& Zhang, Y. 1997, ApJ, 486, 599

Jenkins, E. B., \& Ostriker, J. P. 1991, ApJ, 376, 33

Katz, N., Weinberg, D. H., \& Hernquist, L. 1996, ApJS, 105, 19

Linsky, J. L., Diplas, A., Wood, B. E., Brown, A., Ayres, T. R., \& Savage, B. D. 1995, ApJ, 451, 335

Lynds, C. R. 1971, ApJ, 164, L73
McCullough, P. R. 1992, ApJ, 390, 213

Miralda-Escudé, J., Cen, R., Ostriker, J. P., \& Rauch, M. 1996, ApJ, 471, 582

Miralda-Escudé, J., \& Rees, M. J. 1994, MNRAS, 266, 343

Miralda-Escudé, J., Weinberg, D. H., Hernquist, L., \& Katz, N. 1997, in preparation

Oke, J. B., \& Korycansky, D. G. 1982, ApJ, 255, 11

Persic, M., \& Salucci, P. 1992, MNRAS, 258, L14

Petitjean, P., Mücket, J. P., \& Kates, R. E. 1995, A\&A, 295, L9

Press, W. H., Rybicki, G. B., \& Schneider, D. P. 1993, ApJ, 414, 64 (PRS)

Rauch, M., \& Haehnelt, M. G. 1995, MNRAS, 275, L76

Rauch, M., Haehnelt, M. G., \& Steinmetz, M. 1997a, ApJ, 481, 601

Rauch, M., et al. 1997b, ApJ, 489, 7

Rugers, M., \& Hogan, C. J. 1996a, AJ, 111, 2135 1996b, ApJ, 459, L1

Sargent, W. L. W., Young, P. J., Boksenberg, A., \& Tytler, D. 1980, ApJS, 42,41

Schmidt, M. 1965, ApJ, 141, 1295

Schneider, D. P., Schmidt, M., \& Gunn, J. E. 1994, AJ, 107, 1245

Shapiro, P. R., Giroux, M. L., \& Babul, A. 1994, ApJ, 427, 25

Smette, A., Robertson, J. G., Shaver, P. A., Reimers, D., Wisotzki, L., \& Koehler, T. 1995, A\&AS, 113, 199

Smette, A., Surdej, J., Shaver, P. A., Foltz, C. B., Chaffee, F. H., Weymann, R. J., Williams, R. E., \& Magain, P. 1992, ApJ, 389, 39

Songaila, A., Cowie, L. L., Hogan, C. J., \& Rugers, M. 1994, Nature, 368, 599

Steidel, C. C., \& Sargent, W. L. W. 1987, ApJ, 313, 171

Tytler, D., Fan, X.-M., \& Burles, S. 1996, Nature, 381, 207

Walker, T. P., Steigman, G., Schramm, D. N., Olive, K. A., \& Kang, H.-S. 1991, ApJ, 376, 51

Weinberg, D. H., Hernquist, L., \& Katz, N. 1997, ApJ, 477, 8

Wolfe, A. M., Lanzetta, K. M., Foltz, C. B., \& Chaffee, F. H. 1995, ApJ, 454, 698

Zhang, Y., Anninos, P., \& Norman, M. L. 1995, ApJ, 453, L57

Zhang, Y., Anninos, P., Norman, M. L., \& Meiksin, A. 1997, ApJ, 485, 496 Zuo, L., \& Lu, L. 1993, ApJ, 418, 601 